\title{
Research on the Experimental and the Testing Mode of Highway Bridge Load Capacity based on Static Analysis
}

\author{
Wei Pan \\ Henan Vocational and Technical College of \\ Communications, Zhengzhou city,China, \\ 450000
}

\begin{abstract}
In this paper, we conduct research on the experimental and the testing mode of the highway bridge load capacity based on the static analysis. Modal parameters is refers to the bridge structure calculation of modal frequencies and mode shapes and modal parameters such as the quality. Modal parameters method and other methods differed from the load transverse distribution influence line is composed of the modal parameters are calculated out as every piece of beam deflection; Secondly, according to the modal flexibility from the deformation of beams in the mid-span position of value, according to the deformation value and beam on the number of slices, calculation, draw the influence line of transverse distribution of load. Under this guidance, we enhance the traditional methodologies with the static analysis that will achieve better performance.
\end{abstract}

Keywords- Static Analysis, Load Capacity, Highway Bridge, Testing Mode, Experiment.

\section{Introduction}

With the adjustment of industrial structure, great changes have taken place in transportation, highway traffic in general people's production and life play a very important role. The development of modern freeways, and various kinds of means of transport, marked country highway construction enterprise to a new historical period, bridge is the throat highway

\author{
Tiezeng Zhu \\ Henan Vocational and Technical College of \\ Communications, Zhengzhou city,China, \\ 450000
}

that ensure smooth bridge, to ensure the smooth progress in transportation, therefore, is very important for the evaluation of bridge bearing capacity.

On ultimate bearing capacity calculation of the space steel structure bridges has carried out many studies and put forward the method to evaluate the bearing capacity of single and double layer steel network as a whole. The relevant scholars with reliability theory to study the ultimate bearing capacity of the space steel structure, setting load coefficient, calculate the reliability index, according to the results of the structure bearing capacity coefficient of ensure reliable index value is greater than the engineering setting value. This calculation method for the reliability theory, ultimate bearing capacity of bridge structure evaluation provides important reference basis. The ultimate bearing capacity of the bridge structure evaluation is similar to steel structure evaluation method that need the safety factor method is adopted, therefore, the focus of the ultimate bearing capacity evaluation is load coefficient, determine the elastic and plastic limit of the structure of the bridge assessment method is necessary.

Construction of bridge foundation status quo could be generally summarized as the follows. (1) In bridge construction in the form of pile foundation is relatively common, especially in the lower part of the geology good cases, bridge pile foundation can be upper load transfer to the lower bearing layer, pile foundation types including precast pile, the pre-stressed concrete piles, concrete piles, etc., before two kinds of pile 
can be factory processing in advance, the construction speed, the pile construction technology is relatively complex, but has a good adaptability to different geological conditions. (2) It has a long history of the use of open caisson construction technology, as construction process on the ground ahead of concrete cavity structure, continuous soil inside the cavity depend on the weight of sinking to the design elevation position. (3) The construction of bridge deck. With pile foundation and pile foundation construction of bridge deck are two forms, when the pile embedment depth is shallow, can use direct excavation, soil using the natural slope, when the pile caps is deep embedment depth when they need to take strong measures to the stability of the slope structure [1-3].
In the analysis of the design and structure of the cable-stayed bridge that is necessary to consider the uncertainty of the parameters. In addition, the large span cable-stayed bridge belongs to the flexible structure, geometric nonlinear effect is significant. In stochastic static analysis of cable-stayed bridge, and therefore, we must consider the effect of various geometric nonlinear. Nonlinear mapping and generalization ability, through the sampling techniques, using the deterministic finite element solver, simulated random variables and nonlinear mapping relation between the structural core responses and analyze the variation regularity of structural response of cable-stayed bridge, cable-stayed bridge for random design and structural analysis provides an effective way.
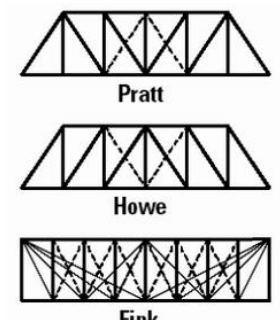

Fink
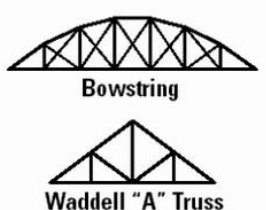
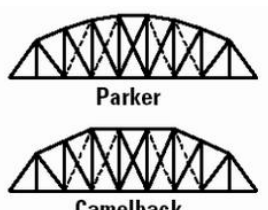

Camelback
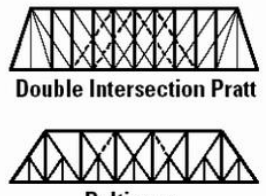

Baltimore

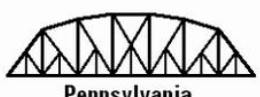

ennsylvania
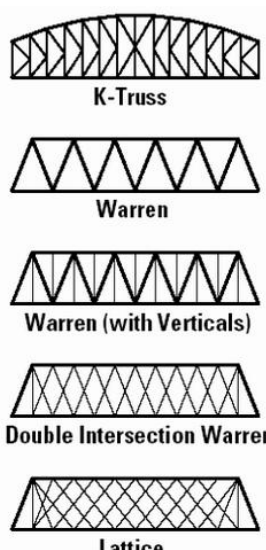

Lattice

Figure 1. The Demonstration of the Highway Bridge Load Patterns

In this paper, we conduct research on the experimental and the testing mode of highway bridge load capacity based on the static analysis. In the above figure one, we illustrate the general highway bridge load patterns. Bridge load test is a practical work, not only requires the staff has a wealth of the field experience, and at the same time need a solid theoretical basis for the guidance. Only the theoretical calculation and the real bridge test fully, to make efforts to detect and obtain satisfactory data, only according to the test results, theoretical calculation model for constantly is likely to make an accurate evaluation. In the following sub-sections, we will discuss the issues in detail.

\section{Our Proposed Methodology}

The Principles of the Static Analysis. Most of the engineering practice, the actual structure of the displacement and the load is a nonlinear relationship as such a system is called nonlinear deformation system. If the system is nonlinear due to the stress and strain relations of nonlinear material, it is called a material nonlinearity, such as material of elastic-plastic, relaxation and creep. If the shape of the structure, the stress of the 
position to make the system that has changed significantly, and can't use the analysis method of linear system is called a geometric nonlinearity, such as the structure of the large deformation and large deflection, etc. There is a class of nonlinear problem is the nonlinear boundary condition, or nonlinear contact, such as various kinds of contact problem [4-5].

For the better analysis, we introduce the neural network for assistance. Neural network consists of two parts, forward and reverse transmission, consists of three layers, respectively for the input layer, hidden layer and output layer, the relationship between the input variables and hidden layer neurons as shown below, where the $h$ represents the hidden neurons.

$$
h=f(a w+b)
$$

Neural network performance function for the network between simulation output and the desired output error sum of squares is demonstrated as the formula two.

$$
E=(t-o)^{T}(t-o)=\sum_{j=1}^{S M}\left(t_{j}-o_{j}\right)^{2}
$$

Material nonlinear problem processing method, usually don't have to modify the whole issue of the expression and just put the linearized stress-strain relationship, solving a series of the linear problems, and through some kind of the correction methods, finally adjust material properties to meet the given constitutive relation, so as to obtain the solution of the problem and structure under the effect of load balance equation is expressed as the follows.

$$
\left(\left[K_{D}\right]+\left[K_{G}\right]\right)\{\delta\}=\{f\}
$$

Algorithm in incremental form gradually applied load, complete balance in every load increment iteration solution to make incremental equilibrium. Before each time solving the NR algorithm to estimate the residual vector, the vector is restoring force load and load by the difference of the load balance and then use a linear model, and test of convergence. If the calculate result does not meet the convergence criterion, to estimate the unbalanced load and modifying stiffness matrix to obtain a new solution. The corresponding balancing equation could be expressed as the follows.

$$
[K T]\left\{D_{u}\right\}=\{F\}-\left\{F_{u r}\right\}
$$

Determined primarily by the linear calculation reaches the yield limit of load value, will this load value as a cut-off point. Cut-off from zero to the process, material plastic deformation does not occur, the linear analysis, step load step into a child from cut-off point to ultimate load value, material plastic deformation took place in this process that will be split, load set multiple sub steps, each time slowly increase a certain load, until the final load values. This method the number of iterations a lot less than the general method, computing speed and improve accordingly [6].

The Stability of the Bridge. Bridges collapsed in force under the action of earthquake happened, in most cases is due to the foundation and basis of severely damage caused by, and this kind of basic circumstance to after the earthquake had the difficulty of the repair of the bridge. Under earthquake force that can appear the foundation soil liquefaction, poor foundation settlement and stability were happening, and the ground will have larger deformation, resulting in the formation appeared different degree of slip and sinking, eventually foundation from being destroyed by the big.

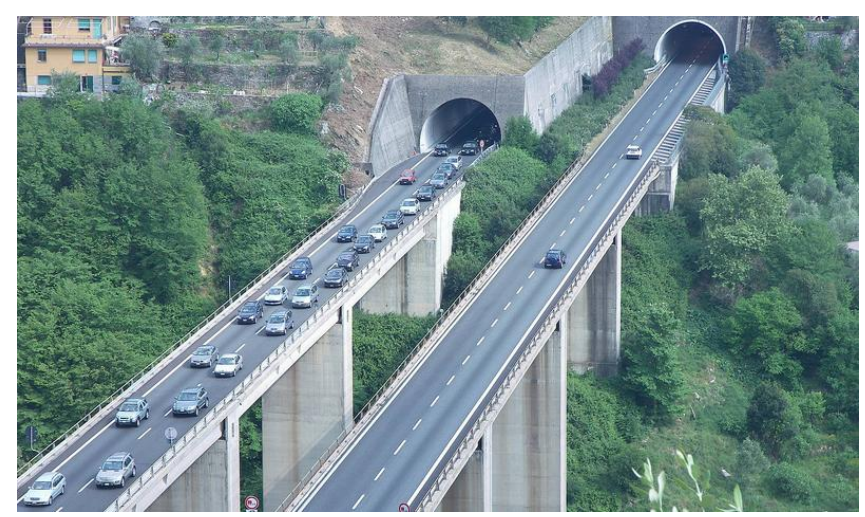

Figure 2. The Stability of the Bridge Illustration 
In order to ensure bridge has good aseismic strength and stability, need of earthquake-resistance ability is higher when design requirements, and to further strengthen research on seismic performance of Bridges, to ensure the bridge project has a good aseismic capacity that can support the earthquake disaster brought by the erosion, fully play the role of the transport hub. (1) Bridge design for the high intensity area shall be installed in vertical, a certain amount of the energy dissipation device, such as using subtract, isolation bearing, and an increase in the joint of girder and pier structure so flexible and damping force and decrease level of load. (2) Simply-supported bridge structure of conventional should strengthen the bridge structure, and to provide adequate to prevent girder reinforcement width displacement of the beam, it also should be the appropriate broaden the width of the pier top beam and bearing, and add to prevent displacement of the basic spacer device. (3) Using the upper structure and bridge to connect fully rigid frame system, and the pile tip through the liquefiable layer to achieve a hard soil layer, due to the increased number of statically indeterminate structure and solid guarantee the bearing capacity of pile tip, to reduce the possibility of failure due to soil deformation [7].

- At present of optical fiber sensors are mainly applied to the bridge detection phase modulation type, used to measure the deformation and internal stress of concrete structure, the detection of large bridge structure, health, etc., one of the most important is the optical fiber sensor is used as a new type of the strain sensor.

- Ultrasonic advantage is high sensitivity, the high speed, low cost, harmless to human body, to positioning and quantitative defect, but the ultrasonic flaw detection of the defects show is not intuitive, flaw detection technology is difficult, easily affected by subjective and objective factors, as well as the inspection result is not easy to preserve, ultrasonic examination of the work surface is smooth, rich experience required inspection personnel to identify thickness of the defect type, suitable for parts inspection, ultrasonic inspection also has its limitations.

- Infrared thermal image detection technology is based on the object's infrared radiation and the internal relations between surface temperature and material properties, with the aid of infrared thermal imager from the object of the infrared radiation into visible images, through analysis of the thermal image characteristics, that intuitive understanding of the surface temperature distribution, thus infer the internal structure of the bridge and surface state.

The Highway Bridge Load Capacity. Bridge structure must bear the moving load when vehicle on bridge deck, structure of the internal force also changes over point on the structure. So you need to research and determine the scope of its change and the change rule and the maximum internal force of the process as the standard design. Therefore, you need to determine is the most unfavorable position and maximum load. As long as after analysis of internal force variation law clearly, other types of loads can be according to the unit under moving load and the structural internal force variation law of superposition principle. Influence line is under the action of the internal force in the mobile unit load caused by the change law of graphics. So, the influence line is the study of vehicle load under moving loads such as maximum bridge structure internal force of the basic tools [8].

Preliminary selected engineering scale and structure of the impact on the surrounding environment type to use requirement, material conditions, construction conditions, factors such as cost, according to the subgrade geological conditions and several kinds of subgrade treatment scheme to consider. 


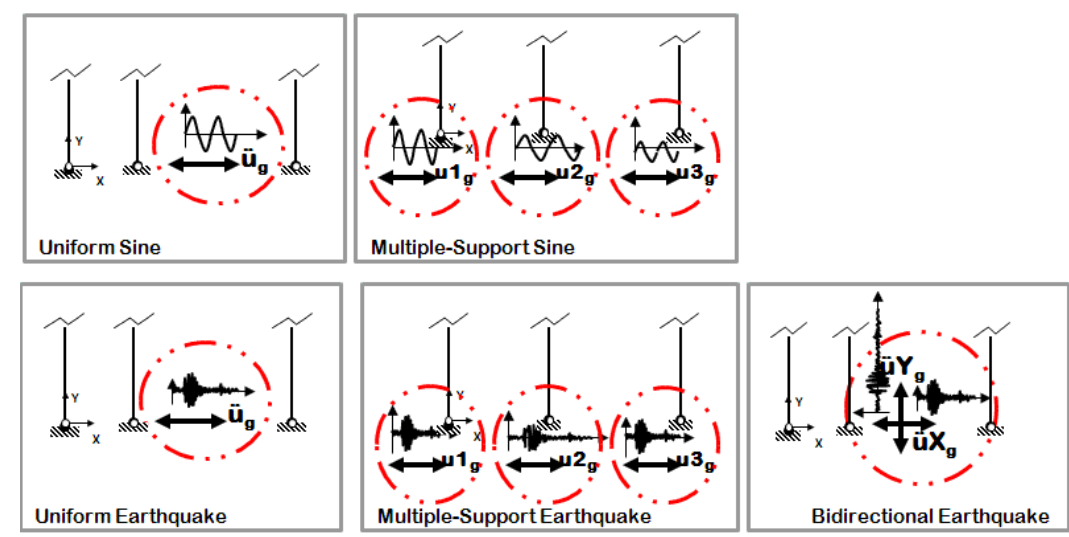

Figure 3. The Verification of the Highway Bridge Load Capacity Simulation

First by the method of dynamic node load, according to bridge finite element model of geometric data, the information such as the locomotive speed moving load into equivalent node load, and then on the basis of Campbell theorem to calculate the covariance matrix of equivalent node load, then to Fourier transform of covariance matrix, the power spectral density matrix of the incentive model. On the basis of power spectral density matrix to study the traffic spatial correlation in the process of incentives, in the case of measuring traffic incentive time domain information, this paper presents a description of the transportation way, without the main properties of the incentive for only based on the output of a bridge system identification has important theoretical significance and value.

\section{Conclusion}

In this paper, we conduct research on the experimental and the testing mode of highway bridge load capacity based on the static analysis. Traffic load are major highway Bridges on the variable load, the investigation is to determine the bridge design load, time-varying reliability analysis both the bridge residual life prediction and calculation of ultimate bearing capacity are the important foundation of the research. The different span simply supported and continuous bridge calculation of vehicle load effect, vehicle load effect of the probability model is established according to the principle of the same probability, have different standard vehicle load effect in span bridge models influence coefficient, on this basis to determine the different vehicle loads subentry coefficients of the structure of the bridge models and relationship models and uses second moment reliability method to calculate the reliability index. In the future, we will combine more mathematical analysis to modify the current model.

\section{Reference}

[1] Campisi, Paul. "Review of Load Rating Highway Bridges: In Accordance with Load and Resistance Factor Rating Method by Lubin Gao." Journal of Bridge Engineering 20.12 (2015): 07515001.

[2] Diaz-Alvarez, Henry, et al. "Structural response of a recycled thermoplastic lumber bridge under civilian and military loads." Journal of Thermoplastic Composite Materials 28.4 (2015): 461-478.

[3] Shu, Jiangpeng. "Structural Analysis of Existing RC Bridge Deck Slabs Structural Analysis of Existing RC Bridge Deck Slabs." (2015).

[4] Matt Laurendeau, A. S. C. E., et al. "Live-Load Testing of a Steel Cantilevered Deck Arched Pratt Truss Bridge." (2015).

[5] Häggström, Jens, et al. "Assessment and full scale failure test of a steel truss bridge." 
IABSE Symposium Report. Vol. 102. No. 9. International Association for Bridge and Structural Engineering, 2014.

[6] Huang, Yufan, et al. "Shaking table tests for the evaluation of the seismic performance of an innovative lightweight bridge with CFST composite truss girder and lattice pier." Engineering Structures 75 (2014): 73-86.

[7] Gavin, Kenneth George, David John Paul Igoe, and Lisa Kirwan. "The effect of ageing on the axial capacity of piles in sand." Proceedings of the Institution of Civil Engineers-Geotechnical Engineering 166.2 (2013): 122-130.

[8] He, Jun, et al. "Experimental investigation of movable hybrid GFRP and concrete bridge deck." Construction and Building Materials 26.1 (2012): 49-64. 